\title{
PLANE DOMAINS WITH HARMONIC INTERPOLATING SEQUENCES WHICH ARE NOT INTERPOLATING SEQUENCES
}

\author{
JUNICHIRO NARITA
}

To the memory of Professor Nobuyuki Suita

Let $D$ be an arbitrary open set in the complex plane $\mathbf{C}$, and $H^{\infty}(D)$ be the set of bounded analytic functions $f(z)$ on $D$, equipped with the supremum norm $\|f\|_{\infty}=\sup _{z \in D}|f(z)|$. A sequence $\left\{z_{j}\right\}$ in $D$ is called an $\left(H^{\infty}(D)\right.$-)interpolating sequence if for all complex valued bounded sequences $\left\{a_{j}\right\}$, the interpolation problem

$$
f\left(z_{j}\right)=a_{j}, \quad j=1,2,3, \ldots
$$

has a solution $f(z)$ in $H^{\infty}(D)$.

For an arbitrary open set $D$ in $\mathbf{C}$, let $h^{\infty}(D)$ be the set of bounded harmonic functions on $D$, equipped with the supremum norm. In the same way as $H^{\infty}(D)$-interpolating sequences, we can consider interpolating sequences for this space $h^{\infty}(D)$. Namely, a sequence $\left\{z_{j}\right\}$ in $D$ is called an $h^{\infty}(D)$-interpolating sequence or harmonic interpolating sequence (in $D$ ), if for any real valued bounded sequence $\left\{a_{j}\right\}$, there exists a function $h \in h^{\infty}(D)$ such that

$$
h\left(z_{j}\right)=a_{j}, \quad j=1,2,3, \ldots
$$

Obviously every interpolating sequence is a harmonic interpolating sequence. For the unit disk $\Delta=\{|z|<1\}$, J. Garnett [7] showed the converse: a harmonic interpolating sequence in $\Delta$ is an interpolating sequence for $H^{\infty}(\Delta)$.

The existence of a plane domain and a sequence which is a harmonic interpolating sequence but not an interpolating sequence was first pointed out by Fisher using a Zalcman domain ([7, p. 191]), and Behrens gives a necessary and sufficient condition for some kind of Zalcman domains to satisfy that a sequence in this domain is an interpolating sequence if and only if it is a harmonic interpolating sequence $([2$, Th. 3.7]). These two results suggest that one typical reason for the existence of a sequence which is a harmonic interpolating sequence, but is not an interpolating sequence, is the existence of a boundary point where the boundary is sufficiently small for bounded analytic functions (in some sense) and is not sufficiently small for bounded harmonic functions (i.e. the point is a regular boundary point in the sense of Dirichlet problem).

2000 Mathematics Subject Classification. 30C85, 30H05, 46J20.

Received March 30, 2004; revised February 3, 2005. 
In this paper, first, we study in this direction and describe two sufficient conditions when harmonic interpolating sequences and interpolating sequences do not coincide (Theorem 2.3, 2.4). Also we give another example of a domain and a sequence, which is also a harmonic interpolating sequence, but not an interpolating sequence, but in this case, this sequence converges to a boundary point where the boundary is sufficiently large even for bounded analytic functions (Theorem 3.1).

\section{Fundamental lemmas}

Thought the proofs of the following two lemmas are essentially included in Fisher's example, we would like to contain these, since we use these lemmas with proof in some detail later.

Lemma 1.1. Let $D$ be a domain in C. Let $\left\{z_{j}\right\}$ be a sequence in $D$, and let $\mu_{j}$ be the harmonic measure relative to $z_{j}$ and $D$. If there exists a sequence of mutually disjoint Borel sets $\left\{F_{j}\right\}$ such that $F_{j} \subset \partial D$ and $\mu_{j}\left(F_{j}\right) \geq \frac{2}{3}(j=1,2, \ldots)$, then $\left\{z_{j}\right\}$ is a harmonic interpolating sequence.

Proof. Let $\left\{a_{j}\right\}$ be a bounded sequence and let $\left\|\left\{a_{j}\right\}\right\|_{\infty}=k$. We set a boundary function $h_{1}$ as

$$
\begin{aligned}
& h_{1}(\zeta)=a_{j} \quad\left(\zeta \in F_{j}\right) \quad(j=1,2, \ldots), \\
& h_{1}(\zeta)=0 \quad\left(\zeta \in \partial D \backslash \bigcup F_{j}\right),
\end{aligned}
$$

and let $f_{1}$ be the solution of Dirichlet problem for $h_{1}$. Then $\left\|f_{1}\right\|_{\infty} \leq k$ and

$$
\begin{aligned}
\left|a_{j}-f_{1}\left(z_{j}\right)\right| & =\left|a_{j}-\int_{\partial D} h_{1} d \mu_{j}\right| \leq \int_{\partial D}\left|a_{j}-h_{1}\right| d \mu_{j}=\int_{\partial D \backslash F_{j}}\left|a_{j}-h_{1}\right| d \mu_{j} \\
& \leq \sup \left|a_{j}-h_{1}\right| \cdot \mu_{j}\left(\partial D \backslash F_{j}\right) \leq 2 k(1-2 / 3)=(2 / 3) k .
\end{aligned}
$$

Hence

$$
\left\|\left\{a_{j}-f_{1}\left(z_{j}\right)\right\}\right\|_{\infty} \leq(2 / 3) k
$$

In the same way as we construct $f_{1}$ from $\left\{a_{j}\right\}$, we can construct $f_{2} \in h^{\infty}(D)$ from $\left\{a_{j}-f_{1}\left(z_{j}\right)\right\}$ such that

$$
\begin{aligned}
\left\|f_{2}\right\|_{\infty} & \leq(2 / 3) k \\
\left|a_{j}-f_{1}\left(z_{j}\right)-f_{2}\left(z_{j}\right)\right| & \leq(2 / 3)^{2} k \quad(j=1,2, \ldots) .
\end{aligned}
$$

Repeating this process, we can take $f_{m} \in h^{\infty}(D)$ satisfying

$$
\begin{gathered}
\left\|f_{m}\right\|_{\infty} \leq(2 / 3)^{m-1} k \quad(m=1,2, \ldots), \\
\left|a_{j}-\sum_{l=1}^{m} f_{l}\left(z_{j}\right)\right| \leq(2 / 3)^{m} k \quad(m=1,2, \ldots ; j=1,2, \ldots) .
\end{gathered}
$$


Then $f=\sum_{m=1}^{\infty} f_{m}$ satisfies

$$
\begin{gathered}
\|f\|_{\infty} \leq \sum_{m=1}^{\infty}(2 / 3)^{m-1} k=3 k, \\
f\left(z_{j}\right)=a_{j} \quad(j=1,2, \ldots) .
\end{gathered}
$$

This implies that the sequence $\left\{z_{j}\right\}$ is a harmonic interpolating sequence.

Lemma 1.2. Let $D$ be a domain in $\mathbf{C}$ and let $\zeta$ be a regular boundary point of $D$ in the sense of Dirichlet problem. If a sequence $S=\left\{z_{n}\right\}$ in $D$ converges to $\zeta$, then $S$ has a subsequence $\left\{z_{n_{j}}\right\}$ which is a harmonic interpolating sequence.

Proof. Let $n_{1}=1$ and let $\mu_{1}$ be the harmonic measure relative to $z_{n_{1}}$ and $D$. For $t>0$, let

$$
E(t)=\{z \in \partial D:|z-\zeta|<t\} .
$$

Since $\mu_{1}(\{\zeta\})=0$, we can take a $t_{1}>0$ such that $\mu_{1}\left(E\left(t_{1}\right)\right)<1 / 6$. We want to take $n_{2}$ to satisfy $\mu_{2}\left(E\left(t_{1}\right)\right)>5 / 6$ where $\mu_{2}$ is the harmonic measure relative to $z_{n_{2}}$. To see this, let $g_{1}$ be a boundary function such that $g_{1}=1$ on $E\left(t_{1}\right)$ and $g_{1}=0$ on $\partial D \backslash E\left(t_{1}\right)$, and let $u_{1}$ be the solution of Dirichlet problem for the boundary values $g_{1}$ in $D$. Since $\zeta$ is a regular boundary point for $D$,

$$
\lim _{D \ni z \rightarrow \zeta} u_{1}(z)=1 \text {. }
$$

Hence we can take $n_{2}$ such that $u_{1}\left(z_{n_{2}}\right)>5 / 6$. Then

$$
u_{1}\left(z_{n_{2}}\right)=\int_{\partial D} g_{1} d \mu_{2}=\int_{E\left(t_{1}\right)} d \mu_{2},
$$

and so $\mu_{2}\left(E\left(t_{1}\right)\right)>5 / 6$.

In a similar manner, we can choose a subsequence $\left\{z_{n_{j}}\right\}$ and a sequence of positive numbers $\left\{t_{j}\right\}$ such that

$$
\begin{aligned}
& t_{1}>t_{2}>\cdots, \quad t_{j} \rightarrow 0 \quad(j \rightarrow \infty), \\
& \mu_{j}\left(E\left(t_{j-1}\right)\right)>5 / 6 \quad(j=2,3, \ldots), \\
& \mu_{j}\left(E\left(t_{j}\right)\right)<1 / 6 \quad(j=1,2, \ldots)
\end{aligned}
$$

where $\mu_{j}$ is the harmonic measure relative to $z_{n_{j}}$. Let

$$
\begin{aligned}
F_{1} & =\partial D \backslash E\left(t_{1}\right), \\
F_{j} & =E\left(t_{j-1}\right) \backslash E\left(t_{j}\right) \quad(j=2,3, \ldots) .
\end{aligned}
$$

Then $\left\{F_{j}\right\}$ are mutually disjoint and satisfies

$$
\mu_{j}\left(F_{j}\right)>2 / 3 \quad(j=1,2, \ldots) .
$$

Hence, by virtue of Lemma 1.1, the subsequence $\left\{z_{n_{j}}\right\}$ is a harmonic interpolating sequence. 


\section{Sequences converging to an irregular boundary point}

A compact set $K$ in $\mathbf{C}$ is called a Painlevé null set if $K$ is a removable singularities for bounded analytic functions, that is, for any open neighborhood $V$ of $K$ and $f \in H^{\infty}(V \backslash K), f$ extends analytically to $V$. It is not difficult to see that $K$ is a Painlevé null set if and only if $H^{\infty}(\mathbf{C} \backslash K)$ consists of only constant functions ([5, p. 86]); the last property is symbolically represented as $\mathbf{C} \backslash K \in O_{A B}$ in the function theory.

Similarly, a compact set $K$ in $\mathbf{C}$ is a removable singularities for bounded harmonic functions if and only if $h^{\infty}(\mathbf{C} \backslash K)$ consists of only constant functions; symbolically $\mathbf{C} \backslash K \in O_{H B}$. The condition $\mathbf{C} \backslash K \in O_{H B}$ is also equivalent to the condition that the logarithmic capacity of $K$ is zero.

It is known that there is a compact set which is a Painlevé null set, but has positive logarithmic capacity ([1, p. 252]). Hence the following Theorem gives another example of plane domains in which interpolating sequences and harmonic interpolating sequences do not coincide.

Theorem 2.1. Let $D$ be an open set in $\mathbf{C}$. Suppose a compact set $K$ in $D$ is a Painleve null set, and the logarithmic capacity of $K$ is positive, then there exists a sequence $S$ in $D \backslash K$ such that $S$ is an $h^{\infty}(D \backslash K)$-interpolating sequence, but not an $H^{\infty}(D \backslash K)$-interpolating sequence.

Proof. It is known that the set of irregular boundary points of an open set has zero capacity ([3, Satz 4.7]). And the Painlevé null set $K$ has no inner points. Therefore, there exists a point $\zeta$ on $K$ such that $\zeta$ is a regular boundary point of $D \backslash K$. By Lemma 1.2, there is a harmonic interpolating sequence $\left\{z_{j}\right\}$ in $D \backslash K$, converging to $\zeta$. On the other hand, $\left\{z_{j}\right\}$ cannot be an $H^{\infty}(D \backslash K)$ interpolating sequence, since any function in $H^{\infty}(D \backslash K)$ extends analytically to $D$.

To state another result concerning harmonic interpolating sequences, we introduce Zalcman domains, which were first studied by Zalcman in [9] and are frequently used in the study of function algebras and the potential theory. Let $\Delta_{0}=\{0<|z|<1\}$ and let $\bar{\Delta}\left(c_{n}, r_{n}\right)=\left\{\left|z-c_{n}\right| \leq r_{n}\right\}$ be a sequence of closed disks with centers on the positive real axis satisfying

$$
\begin{aligned}
& \bar{\Delta}\left(c_{n}, r_{n}\right) \subset \Delta_{0} \quad(n=1,2, \ldots), \\
& \bar{\Delta}\left(c_{n}, r_{n}\right) \cap \bar{\Delta}\left(c_{m}, r_{m}\right)=\emptyset \quad(n \neq m), \\
& c_{1}>c_{2}>\cdots, \quad c_{n} \rightarrow 0 \quad(n \rightarrow \infty) .
\end{aligned}
$$

Then the domain $\Delta_{0} \backslash \bigcup_{n=1}^{\infty} \bar{\Delta}\left(c_{n}, r_{n}\right)$ is called a Zalcman domain.

Now we recall the Fisher's example. Zalcman showed in [9], that when $\sum_{n=1}^{\infty} r_{n} / c_{n}<\infty$, any bounded analytic function $f$ on the Zalcman domain $D=$ $\Delta_{0} \backslash \bigcup_{n=1}^{\infty} \bar{\Delta}\left(c_{n}, r_{n}\right)$ has a limit 


$$
\lim _{\mathbf{R}^{-} \ni z \rightarrow 0} f(z)
$$

where $\mathbf{R}^{-}$represents the negative real axis. Therefore, any sequence tending 0 along negative real axis cannot be an $H^{\infty}(D)$-interpolating sequence. Fix two constants $a, b$ such as $0<b<a<1$, and set

$$
c_{n}=a^{n}, \quad r_{n}=b^{n} \quad(n=1,2, \ldots) .
$$

Then $\sum_{n=1}^{\infty} r_{n} / c_{n}=\sum_{n=1}^{\infty}(b / a)^{n}<\infty$. We remark that, though finite closed disks $\bar{\Delta}\left(a^{n}, b^{n}\right)$ may intersect, we can choose an integer $N$ such that $R=\Delta_{0} \backslash \bigcup_{n=N}^{\infty}$ $\bar{\Delta}\left(a^{n}, b^{n}\right)$ is a Zalcman domain (for example, take $N$ such as $b^{N}<\left(a^{N}-a^{N+1}\right) / 2$ $\left.=a^{N}(1-a) / 2\right)$. Let

$$
A_{n}=\partial R \cap\left\{a^{n+1 / 2} \leq|z| \leq a^{n-1 / 2}\right\},
$$

and let $\operatorname{cap}\left(A_{n}\right)$ be the logarithmic capacity of $A_{n}$. For $n \geq N, A_{n}=\partial \Delta\left(a_{n}, b_{n}\right)$. Since the logarithmic capacity of a disk of radius $r$ is $r$, we have $\operatorname{cap}\left(A_{n}\right)=b^{n}$. Hence the series of the Wiener's criterion ([4], [8, p. 104]) diverges as

$$
\sum_{n=N}^{\infty} \frac{n}{\log \left(1 / \operatorname{cap}\left(A_{n}\right)\right)}=\sum_{n=N}^{\infty} \frac{n}{-n \log b}=\sum_{n=N}^{\infty} \frac{1}{-\log b}=\infty .
$$

This implies that the boundary $\partial R$ is thick at the origin in the sense of fine topology, or equivalently, the origin is a regular boundary point for $R$. From Lemma 1.2 , there exists a sequence $\left\{z_{j}\right\}$ on negative real axis such that $\left\{z_{j}\right\}$ is a harmonic interpolating sequence.

Though Fisher's example is not contained in the result of Theorem 2.1, it depends on the fact that in some sense the boundary of the Zalcman domain is small for bounded analytic functions but not small for bounded harmonic functions at the origin. By virtue of the study of Gamelin and Garnett in [6], we can consider this phenomena in more general domains.

Since $H^{\infty}(D)$ is a Banach algebra with the supremum norm, we can consider the maximal ideal space $\mathscr{M}(D)$ of $H^{\infty}(D)$. It consists of all nonzero complex valued multiplicative linear functionals, called complex homomorphisms, on $H^{\infty}(D)$. By the weak star topology inherited from the dual space $H^{\infty}(D)^{*}$ of $H^{\infty}(D), \mathscr{M}(D)$ is a compact Hausdorff space.

When $D$ is a bounded open set in $\mathbf{C}$, the coordinate function $Z$ belongs to $H^{\infty}(D)$. The Gelfand transform $\hat{Z}$ maps $\mathscr{M}(D)$ onto $\bar{D}$, and $\left.\hat{Z}^{-1}\right|_{D}$ gives a homeomorphism of $D$ onto the open subset $\hat{Z}^{-1}(D)$ of $\mathscr{M}(D)$, which coincides with the identification by the point evaluations ([5, p. 2]).

When $D$ is unbounded open set in $\mathbf{C}$, the usual coordinate function does not belong to $H^{\infty}(D)$. However, if $H^{\infty}(D)$ contains a nonconstant function, there exists a continuous function $\Upsilon$ from $\mathscr{M}(D)$ onto $\bar{D} \subset \hat{\mathbf{C}}$, such that $\left.\Upsilon^{-1}\right|_{D}$ gives a homeomorphism of $D$ onto the open subset $\Upsilon^{-1}(D)$ of $\mathscr{M}(D)$, and this coincides with the identification by the point evaluations. ([5, p. 87]).

For a bounded open set $D$ in $\mathbf{C}$ and a point $\zeta$ in $\bar{D}$, the fiber $\mathscr{M}_{\zeta}(D)$ is defined by $\mathscr{M}_{\zeta}(D)=\hat{Z}^{-1}(\zeta)$. When $D$ is not bounded and $\mathbf{C} \backslash D$ is not a Painlevé 
null set, we can replace $Z$ by $\Upsilon$, and set $\mathscr{M}_{\zeta}(D)=\Upsilon^{-1}(\zeta)$. According to usual terminology, $\mathscr{M}_{\zeta}(D)$ is called a peak set if there exists an $f \in H^{\infty}(D)$ such that $\hat{f}=1$ on $\mathscr{M}_{\zeta}(D)$ and $|\hat{f}|<1$ on $\mathscr{M}(D) \backslash \mathscr{M}_{\zeta}(D)$.

Theorem 2.2. Let $D$ be an open set in $\mathbf{C}$. Suppose there is a point $\zeta$ such that $\zeta$ is a regular boundary point for $D$ in the sense of Dirichlet problem, and the fiber $\mathscr{M}_{\zeta}(D)$ is not a peak set. Then there exists a sequence $S$ in $D$ such that $S$ is an $h^{\infty}(D)$-interpolating sequence, but not an $H^{\infty}(D)$-interpolating sequence.

Proof. When $\mathscr{M}_{\zeta}(D)$ is not a peak set, there exists a sequence $\left\{z_{j}\right\}$ in $D$ such that $\left\{z_{j}\right\}$ converges to a point $\psi$ (called a distinguished homomorphism) in $\mathscr{M}_{\zeta}(D)$ in the norm of the dual space $\left(H^{\infty}(D)\right)^{*}([6$, Section 4]). This means that any subsequence of $\left\{z_{j}\right\}$ is not an $H^{\infty}(D)$-interpolating sequence. On the other hand, by Lemma 1.2 , we can take a subsequence $S=\left\{z_{j_{k}}\right\}$ such that $S$ is an $h^{\infty}(D)$-interpolating sequence.

\section{Sequences converging to a regular boundary point}

Considering Theorem 2.1 and Theorem 2.2, we might expect that the existence of these kinds of boundary point is a necessary and sufficient condition for the existence of a sequence which is a harmonic interpolating sequence but not an interpolating sequence. However, an example, which we will construct, shows that this is not true.

To this purpose, we consider domains $D=\Delta \backslash \bigcup_{n=1}^{\infty} \bar{\Delta}\left(c_{n}, r_{n}\right)$, similar to Zalcman domains, but satisfy

$$
c_{1}<c_{2}<\cdots, \quad c_{n} \rightarrow 1 \quad(n \rightarrow \infty)
$$

instead of (3). We call these domains as boundary Zalcman domains. Note that the boundary $\partial D$ of a boundary Zalcman domain $D$ is large enough for bounded analytic functions at any boundary point $\zeta \in \partial D$; in fact, it is easy to see that the fiber $\mathscr{M}_{\zeta}(D)$ is a peak set.

THEOREM 3.1. There exists a boundary Zalcman domain $D$ and a sequence $S$ in $D$ such that $S$ is a harmonic interpolating sequence but not an interpolating sequence.

Proof. Let $R=\Delta_{0} \backslash \bigcup_{n-1}^{\infty} \bar{\Delta}\left(c_{n}, r_{n}\right)$ be a Zalcman domain of Fisher's example, and let $\left\{z_{j}\right\}$ be a sequence in $R$ such that $\left\{z_{j}\right\}$ is a harmonic interpolating sequence but not an interpolating sequence. We can assume that the sequence $\left\{z_{j}\right\}$ is constructed as in the proof of Lemma 1.2, and that there exist mutually disjoint subsets $F_{j} \subset \partial R$ such that $\mu_{j}\left(F_{j}\right)>2 / 3(j=1,2, \ldots)$ where $\mu_{j}$ is the harmonic measure relative to $z_{j}$. By deleting $z_{1}$ and renumbering, we can also assume that

$$
F_{j} \subset \bigcup_{n=1}^{\infty} \partial \Delta\left(c_{n}, r_{n}\right) \quad(j=1,2, \ldots) .
$$


For each positive integer $n$, there exists an positive integer $N(n)$ such that

$$
\bigcup_{j=1}^{n} F_{j} \subset \bigcup_{m=1}^{N(n)} \partial \Delta\left(c_{m}, r_{m}\right) .
$$

Let $\left\{V_{n}\right\}$ be a sequence of mutually disjoint closed disks in $\Delta$, whose centers are on the positive real axis, and converge to $1 \in \partial \Delta$. And in each $V_{n}$, we take $N(n)$ mutually disjoint disks

$$
\bar{\Delta}\left(c_{n, k}, r_{n, k}\right) \subset V_{n} \quad\left(c_{n, k}>0, r_{n, k}>0\right) \quad(k=1,2, \ldots, N(n))
$$

such that $V_{n} \backslash \bigcup_{n=1}^{N(n)} \bar{\Delta}\left(c_{n, k}, r_{n, k}\right)$ is similar to $\Delta \backslash \bigcup_{n=1}^{N(n)} \bar{\Delta}\left(c_{k}, r_{k}\right)$. We denote this similar transformation by $\varphi_{n}$ :

$$
\varphi_{n}\left(\Delta \backslash \bigcup_{n=1}^{N(n)} \bar{\Delta}\left(c_{k}, r_{k}\right)\right)=V_{n} \backslash \bigcup_{n=1}^{N(n)} \bar{\Delta}\left(c_{n, k}, r_{n, k}\right),
$$

and let

$$
\begin{gathered}
W=\Delta \backslash \bigcup_{n=1}^{\infty} \bigcup_{k=1}^{N(n)} \bar{\Delta}\left(c_{n, k}, r_{n, k}\right), \\
w_{n, j}=\varphi_{n}\left(z_{j}\right) \quad(j=1,2, \ldots, n ; n=1,2, \ldots) .
\end{gathered}
$$

We show that the boundary Zalcman domain $W$ and the sequence

$$
S=\left\{w_{n, j}: j=1,2, \ldots, n ; n=1,2, \ldots\right\}
$$

in $W$ give the desired example.

Let

$$
\tilde{F}_{n, j}=\varphi_{n}\left(F_{j}\right) \quad(j=1,2, \ldots, n ; n=1,2, \ldots) .
$$

Then the sequence of subsets of $\partial W$,

$$
\left\{\tilde{F}_{n, j}: j=1,2, \ldots, n ; n=1,2, \ldots\right\}
$$

are mutually disjoint, and for the harmonic measure $\tilde{\mu}_{n, j}$ relative to $w_{n, j}$ and $W$, we have

$$
\tilde{\mu}_{n, j}\left(\tilde{F}_{n, j}\right)>\mu_{j}\left(F_{j}\right)>2 / 3 .
$$

Hence, by Lemma $1.1, S$ is a harmonic interpolating sequence in $W$.

Suppose $S$ is an interpolating sequence in $W$. Then for any bounded sequence $\left\{a_{j}\right\} \in l^{\infty}$, there is a function $\tilde{f} \in H^{\infty}(W)$ such that

$$
\tilde{f}\left(w_{n, j}\right)=a_{j} \quad(j=1,2, \ldots, n ; n=1,2, \ldots) .
$$

Let

$$
f_{n}=\tilde{f} \circ \varphi_{n} \quad(n=1,2, \ldots) .
$$

Then $f_{n} \in H^{\infty}(R),\left\|f_{n}\right\|_{\infty} \leq\|\tilde{f}\|_{\infty}(n=1,2, \ldots)$, and 


$$
f_{n}\left(z_{j}\right)=\tilde{f} \circ \varphi_{n}\left(z_{j}\right)=\tilde{f}\left(w_{n, j}\right)=a_{j} \quad(j=1,2, \ldots, n ; n=1,2, \ldots) .
$$

By taking uniform convergent limit of a subsequence of $\left\{f_{j}\right\}$, we have a function $f \in H^{\infty}(R)$ such that

$$
f\left(z_{j}\right)=a_{j} \quad(j=1,2, \ldots) .
$$

This contradicts the hypothesis of $R$ and $\left\{z_{j}\right\}$.

\section{REFERENCES}

[ 1 ] L. Ahlfors and L. Sario, Riemann surfaces, Princeton Univ. Press, Princeton, N. J., 1960.

[ 2 ] M. Behrens, Interpolation and Gleason parts in L-domains, Trans. Amer. Math. Soc. 286 (1984), 203-225.

[ 3 ] C. Constantinescu And A. CoRnea, Ideale Ränder Riemannscher Flächen, Springer-Verlag, Berlin, 1963.

[4] T. GAmelin AND H. Rossi, Jensen measures and algebras of analytic functions, in Function algebras, F. Birtel (ed.), Scott, Foresman and Co., 1966, 13-35.

[5] T. Gamelin, Lectures on $H^{\infty}(D)$, Univ. Nacional de la Plata, Argentine, 1972.

[6] T. Gamelin and J. Garnett, Distinguished homomorphisms and fiber algebras, Amer. J. Math. 92 (1970), 455-474.

[ 7 ] J. GarnetT, Interpolating sequences for bounded harmonic functions, Indiana Univ. Math. J. 21 (1971), 187-192.

[8] M. Tsusi, Potential theory in modern function theory, Maruzen, Tokyo, 1959.

[9] L. Zalcman, Bounded analytic functions on domains of infinite connectivity, Trans. Amer. Math. Soc. 144 (1969), 241-270.

DePartment of Mathematics

Daido InStitute of TeChNOLOGY

TAKiHARU, Minami, NAgOYa 457-8530

JAPAN

e-mail: narita@daido-it.ac.jp 